\title{
PERFIL DA VIOLÊNCIA CONTRA CRIANÇAS EM UMA CAPITAL BRASILEIRA
}

Profile of violence against children in a brazilian capital

Perfil de la violencia contra los niños en una capital brasileña Sousa Silva Santos ${ }^{1}$, Leonora Rezende Pacheco ${ }^{2}$, Juliana Bastoni da Silva ${ }^{1}$, Cintia Flôres Mutti $^{3}$

${ }^{1}$ Grupo de Estudos e Pesquisas em Saúde da Criança (GEPESC-UFT), Curso de Enfermagem, Universidade Federal do Tocantins, Palmas-TO, Brasil.

${ }^{2}$ Grupo de Estudos e Pesquisas em Saúde da Criança (GEPESC-UFT), Curso de Enfermagem, Universidade Federal de Goiás, Goiânia-GO, Brasil.

${ }^{3}$ Grupo de Estudos e Pesquisas em Saúde da Criança (GEPESC-UFT), Colégio Politécnico, Universidade Federal de Santa Maria, Santa Maria-RS, Brasil.

*Correspondência: Laboratório 4, Sala 2, Av. NS 15, 109 Norte, Palmas, Tocantins, Brasil. CEP:77.010-090. emailleidienesantos@uft.edu.br.

\section{RESUMO}

A violência está presente nos diversos cenários sociais e a infantil, especificamente, provoca repercussões negativas ao longo da vida do indivíduo, configurando-se em desafio das políticas públicas de saúde mundialmente. Nesse contexto, objetivou-se identificar o perfil da violência infantil no município de Palmas, Tocantins, Brasil, por meio de pesquisa documental e análise descritiva dos casos de violência infantil registrados no Sistema de Informação de Agravos de Notificação no período de 2009 a 2015. Ao total, foram notificados $737(100 \%)$ casos de violência contra crianças, sendo possível identificar um expressivo aumento do número de registros ao longo dos anos, alcançando a marca de $268(36,4 \%)$ somente em 2015. Acredita-se que conhecer o perfil da violência infantil pode contribuir para seu enfrentamento e fortalecimento da rede de proteção da criança.

Palavras-chave: Maus-Tratos Infantis; Violência; Violência Doméstica.

\begin{abstract}
Violence is present in various social scenarios, and child specifically causes negative repercussions throughout the life of the individual, constituting challenges of public health policies worldwide. In this context, the objective was to identify the profile of child violence in the city of Palmas, Tocantins, Brazil, by means of documentary research and descriptive analysis of cases of child violence registered in the Reporting Disease Information System during 2009 to 2015. In total, 737 (100\%) cases of violence against children were reported, and it was possible to identify a significant increase in the number of records over the years, reaching $268(36.4 \%)$ in 2015 alone. It is believed that knowing the profile of child violence can contribute to its confrontation and strengthening of the child protection network.
\end{abstract}

Keywords: Child Abuse; Violence; Domestic Violence.

\section{RESUMEN}

La violencia está presente en varios escenarios sociales, la violencia contra el niño específicamente provoca repercusiones negativas a lo largo de la vida del individuo, lo que constituye un desafío para las políticas de salud pública en todo el mundo. En este contexto, el objetivo fue identificar el perfil de violencia infantil en la ciudad de Palmas, Tocantins, Brasil, mediante la investigación documental y el análisis descriptivo de los casos de violencia infantil registrados en Notificación de trastornos de la información durante el período. De 2009 a 2015. En total, 
se notificaron 737 (100\%) casos de violencia contra niños, y fue posible identificar un aumento significativo en el número de registros a lo largo de los años, alcanzando la marca de 268 (36,4\%) solo en 2015. Conocer el perfil de la violencia infantil puede contribuir a su confrontación y fortalecimiento de la red de protección infantil.

Descriptores: Maltrato Infantil; La Violencia; La Violencia Doméstica.

\section{INTRODUÇÃO}

No cenário nacional, diversos avanços foram alcançados nas áreas de promoção e proteção dos direitos das crianças ao longo dos últimos anos, como a redução da mortalidade infantil e da desnutrição, e a ampliação do acesso aos serviços de saúde e cobertura vacinal. Entretanto, emergiram outros desafios como a estagnação dos índices de mortalidade neonatal e a incidência aumentada de prematuridade, de condições crônicas (BRANQUINHO et al., 2018) e dos casos de violência (BRASIL, 2014).

Considerando especificamente a agressão infantil, é válido pontuar que esse agravo à saúde, apesar de comprovadamente repercutir negativamente na vida da criança (PLATT et al., 2018; TURNER et al., 2018), apresenta números expressivos em todo o mundo e configura-se em fenômeno culturalmente enraizado e aceito em muitas sociedades (UNICEF, 2017).

Para se ter ideia, somente no Brasil foram notificados mais de 30 mil casos de agressão contra menores de 10 anos em 2014 (BRASIL, 2014), sendo que o quantitativo de registros representa somente a ponta do iceberg das violências cotidianas que efetivamente acontecem. Sob esse quantitativo visível, existem inúmeros maus-tratos que não alcançam visibilidade social (SANTOS et al., 2019; RIBEIRO et al., 2015).

Nesse cenário, apresenta-se como dificuldade e, ao mesmo tempo, maior desafio, construir redes coordenadas e sistematizadas que priorizem as medidas preventivas, pois o enfrentamento das situações de violência demanda articulação conjunta e efetiva com os diversos setores e atores (EGRY et al., 2018).

Além disso, é importante quantificar e caracterizar as formas de violência que acometem os menores de idade, uma vez que a condução da ação pelos profissionais responsáveis pelo atendimento à criança maltratada deve ser feita de maneira particular, respeitando a individualidade de cada caso (NUNES e SALES, 2016).

Nesse sentido, essa pesquisa objetivou identificar o perfil da violência infantil no município de Palmas, Tocantins, Brasil, pois acredita-se que conhecer as características das agressões pode contribuir para implementação de ações pelos diversos atores envolvidos na rede de proteção à criança que, efetivamente, contribuam para redução desse agravo à saúde.

\section{METODOLOGIA}

Pesquisa documental, realizada por meio da análise descritiva dos casos de violência infantil ocorridos no município de Palmas, Tocantins, Brasil, registrados no SINAN Net (Sistema de Informação de Agravos de Notificação) no período de 2009 a 2015.

No mês de janeiro de 2016, informações das "fichas de notificação/investigação individual de violência doméstica, sexual e/ou outras violências interpessoais" foram disponibilizadas às pesquisadoras em planilha eletrônica pelo Centro de Informações Estratégicas de Vigilância em Saúde (CIEVS) da Secretaria Municipal de Saúde.

O CIEVS tem por finalidade fomentar a captação de notificações, a análise epidemiológica dos dados e dar respostas oportunas às emergências 
em saúde pública de relevância nacional e estadual (BRASIL, 2007).

De fevereiro a março/2016, as informações foram digitadas em um banco de dados próprio, codificados e submetidos à análise estatística descritiva, por meio de frequência absoluta e relativa. As variáveis analisadas foram: idade, sexo, tipo de violência, local da ocorrência, ano da ocorrência e vínculo com o agressor.

Considerando que o SINAN Net do município de Palmas foi implantado inicialmente em 2006, com pleno funcionamento a partir de 2009 , adotou-se o seguinte critério de inclusão para essa pesquisa: "fichas de notificação/investigação individual de violência doméstica, sexual e/ou outras violências interpessoais" registradas nos anos de 2009 a 2015, em que os casos tenham ocorrido no referido município e que a criança em situação de violência apresentasse idade de até 12 anos. Foram excluídas as fichas em que não constava o registro da variável "idade da vítima".

Esta pesquisa foi aprovada pela Secretaria Municipal de Saúde de Palmas, DIEP Nº 254/2015, e por Comitê de Ética em Pesquisa com Seres Humanos, CAAE No 1.337.178.

\section{RESULTADOS}

No município de Palmas, de 2009 a 2015, foram notificados $737(100 \%)$ casos de violência contra crianças, sendo possível identificar um expressivo aumento do número de registros ao longo dos anos, alcançando a marca de $521(70,7 \%)$ em 2014 e 2015.

Figura 1. Total de casos de violência contra crianças de até doze anos notificados de 2009 a 2015, conforme ano do registro. Palmas, Tocantins, Brasil, 2016, $n=737$

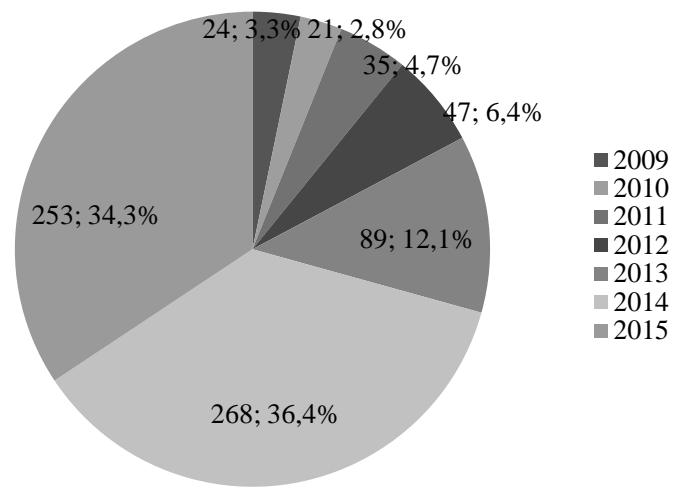

Do total de 737 (100\%) notificações de violência contra crianças, em 328 (44,5\%) não houve registro do tipo ocorrido, esse campo da ficha permaneceu em branco ou constava a opção "outros", sem a exata descrição. Nas demais (409; 55,5\%), foi registrado o total de 645 (100\%) tipos de violência. Sendo assim, contatou-se que muitas crianças foram expostas a mais de uma agressão, com maior frequência para as de ordem sexual $(247 ; 38,3 \%)$, física $(199 ; 30,8 \%)$ e psicológica $(138 ; 21,4 \%)$ (Figura 2).

Figura 2. Total de violências registradas contra a criança de até doze anos notificados de 2009 a 2015, conforme tipo de violência. Palmas, Tocantins, Brasil, 2016, $\mathrm{n}=645$

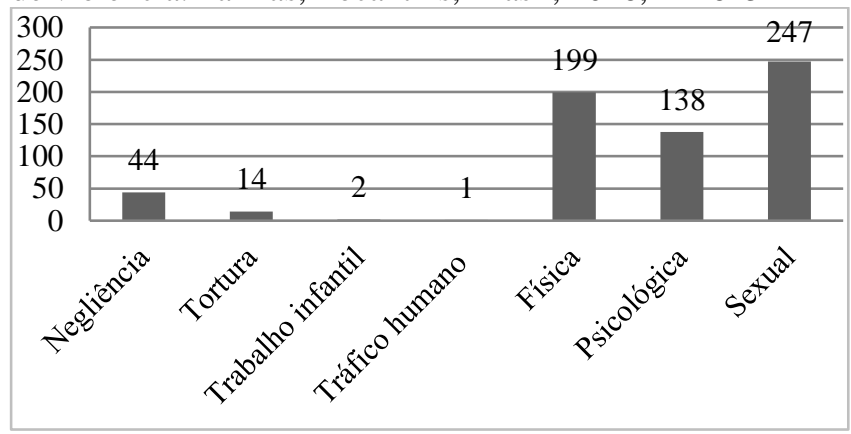

Em relação à faixa etária, identificou-se que a idade de onze a doze alcançou maior frequência de ocorrência nas violências físicas $(52 ; 26,1 \%)$, psicológicas $(42 ; 30,4 \%)$ e sexuais $(64 ; 25,9 \%)$. Já a negligencia acometeu principalmente crianças menores, com até cinco anos $(33 ; 75 \%)$. Nota-se, assim, que no município a violência acomete crianças de diversas idades, em especial aquelas com onze a doze anos, sendo que essa faixa etária representou 
mais de 30\% (223) do total de notificações realizadas de 2009 a 2015 (Figura 3).

Figura 3. Total de violências registradas contra a criança de até doze anos notificados de 2009 a 2015, conforme faixa etária. Palmas, Tocantins, Brasil, 2016, $\mathrm{n}=737$

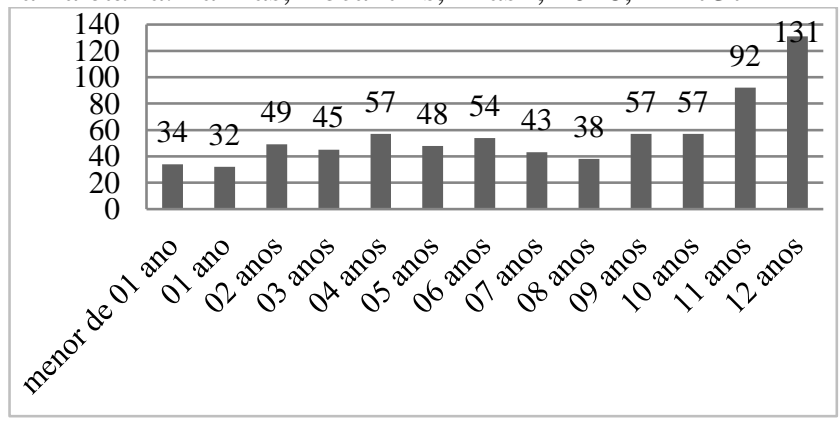

A partir das $645(100 \%)$ fichas em que houve registro do sexo da criança, identificou-se que a maioria dos casos de violência ocorreu em meninas (471; 73\%), e que para ambos os sexos os principais tipos de agressão foram a sexual, física e psicológica (Figura 4).

Figura 4. Total de violências registradas contra a criança de até doze anos notificados de 2009 a 2015, conforme tipo de violência e sexo. Palmas, Tocantins, Brasil, 2016, n = 645

\begin{tabular}{lccc}
\hline \multirow{2}{*}{ Tipo de violência } & $\mathbf{f}(\%)$ & \multicolumn{2}{c}{ Sexo } \\
\cline { 3 - 4 } Sexual & Masculino f(\%) & $\begin{array}{c}\text { Feminino } \\
\mathbf{f}(\%)\end{array}$ \\
\hline Física & $199(100 \%)$ & $74(37,1 \%)$ & $125(62,8 \%)$ \\
\hline Psicológica & $138(100 \%)$ & $37(26,8 \%)$ & $101(73,1 \%)$ \\
\hline Negligência & $44(100 \%)$ & $19(43,1 \%)$ & $25(56,8 \%)$ \\
\hline Tortura & $14(100 \%)$ & $9(64,2 \%)$ & $5(35,7 \%)$ \\
Trabalho infantil & $2(100 \%)$ & $0(0 \%)$ & $2(100 \%)$ \\
\hline Tráfico humano & $1(100 \%)$ & $0(0 \%)$ & $1(100 \%)$ \\
\hline
\end{tabular}

Os registros apontam, também, a própria residência da criança como o principal ambiente em que a agressão acontece $(294 ; 85,7 \%)$, embora seja importante resgatar que menos da metade do total de fichas $(343 ; 46,5 \%)$ estava preenchida com a respectiva informação.

De modo similar, somente em 308 (41,8\%) fichas houve registro do tipo de vínculo/grau de parentesco do agressor com a criança. Considerando esses dados, pessoas que fazem parte do convívio da criança, tais como pai $(61 ; 19,8 \%)$, padrasto (46; $14,9 \%)$ e mãe $(39 ; 12,7 \%)$, foram apontadas como as principais perpetradoras da violência infantil (Figura 5).

Figura 5. Total de violências registradas contra a criança de até doze anos notificados de 2009 a 2015, conforme tipo vínculo/grau de parentesco do agressor. Palmas, Tocantins, Brasil, 2016, $\mathrm{n}=308$

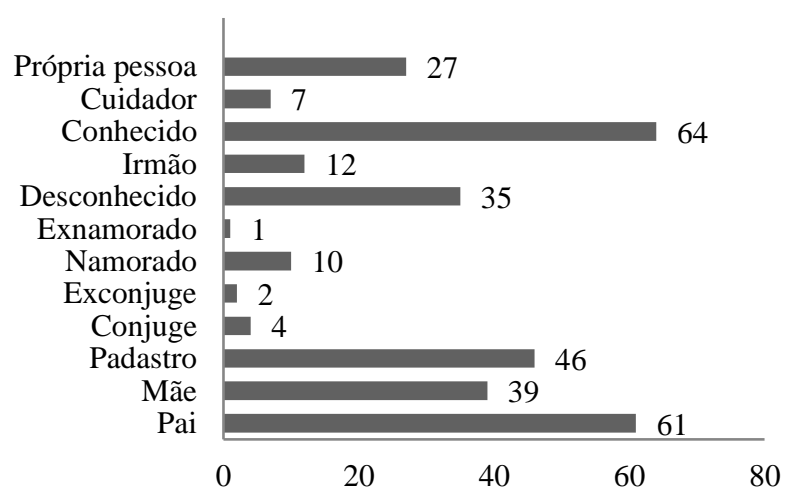

\section{DISCUSSÃO}

Assim como identificado nesta pesquisa, diversos países tem apresentado aumento dos registros de violência contra a criança nos últimos anos. Estima-se que a cada sete minutos, em algum lugar do mundo, uma criança ou adolescente, com idade de 10 a 19 anos, seja morto, vítima de homicídio ou de algum tipo de conflito armado ou violência coletiva. Somente em 2015 foram vitimados mais de 80 mil meninos e meninas na referida faixa etária, sendo que 24,5 mil dessas mortes aconteceram na região da América Latina e do Caribe (UNICEF, 2017).

Percebe-se, desse modo, que o enfrentamento da violência mantém-se como desafio atual, especialmente por tratar-se de um fenômeno complexo, que envolve aspectos culturais, econômicos e sociais (MINAYO et al., 2018; LIRA et al., 2018). 
A violência acomete crianças de diversas faixas etárias e pode diferir substancialmente por categoria de maus tratos, gênero e local em que ocorre (MOODY et al., 2018; UNICEF et al., 2017).

Nesse contexto, pontua-se que é importante traçar o perfil desse agravo à saúde nos diversos cenários, de modo a possibilitar implementação de estratégias que efetivamente corroborem sua redução (UNICEF, 2017).

No município de Palmas, bem como em outras regiões e países, os casos de agressão sexual são os mais prevalentes nas crianças, especialmente no sexo feminino (MOODY et al., 2018; UNICEF, 2017). Destaca-se que o abuso sexual fere a individualidade pela invasão agressiva, roubando da infância a "mágica" que lhe é pertinente e necessária, comprometendo o viver na infância e para além dela. Sendo assim, são urgentes ações que interrompam o ciclo de violência sexual em crianças (SENA et al., 2018), de modo a favorecer que elas tenham a oportunidade de alcançar o crescimento e desenvolvimento plenos.

Colabora para o enfrentamento da violência contra criança que os diversos atores envolvidos na rede de proteção desse público realizem diálogo permanente, articulação de vontades e capacitação permanente para o trabalho coletivo e intersetorial (EGRY et al., 2018; DESLANDES et al., 2016).

Pessoas conhecidas e a família, em especial os pais, apareceram como principais perpetradores da violência infantil neste estudo, fato também identificado em outros locais do país (PLATT et al., 2018; NUNES e SALES, 2016) e no exterior (UNICEF, 2017).

A família, que deveria exercer o papel de proteger a criança, constitui um dos principais provedores de agressões, comprometendo-lhe o bemestar e o desempenho psicossocial (PLATT et al., 2018; NAKAYA et al., 2016). Percebe-se que a violência intrafamiliar é uma realidade alarmante e representa agravo de difícil intervenção, visto que emerge de uma complexa combinação de fatores (SCHEK et al., 2016).

Nesse contexto, a falta de medidas que efetivamente protejam as crianças pode corroborar a manutenção do ciclo de violência infantil. Para se ter ideia, somente $9 \%$ dos menores de 5 anos vivem em países em que a punição corporal é totalmente proibida, deixando cerca de 607 milhões de crianças pequenas sem proteção legal completa (UNICEF, 2017).

Considerando-se que experiências adversas durante a infância podem repercutir negativamente na vida adulta (AFIFI et al., 2019; AUSTIN, 2018), é indispensável às famílias e sociedade que a violência infantil seja entendida como uma forma grave de desrespeito aos direitos humanos fundamentais. Assim, é imprescindível superar o paradigma de que o maltrato infantil seja ação natural ou apenas um modo particular de os pais lidarem com os seus filhos e mudar a realidade em que a violência seja fenômeno socialmente aceito e praticado (SANTOS et al., 2019; NUNES e SALES, 2016).

São urgentes ações que corroborem com a formação qualificada dos profissionais que se deparam com as situações de violência infantil nas diversas áreas, no que se refere aos processos de trabalho, cuidados e medidas específicas que realizam, para despertar e estimular intervenções apropriadas ao momento do ciclo de violência, podendo inclusive levar à sua interrupção (BERTHOLD et al., 2019; EGRY et al., 2017).

Assim, atuar efetivamente no enfrentamento das violências exige sensibilidade, conhecimento do problema, do contexto e atuação integrada para viabilizar momentos de reflexão, partilha de experiências e aprendizado (LIRA et al., 2018). Sugere-se, como estratégia para melhorar a confiança 
do profissional e competência para atuar na rede de proteção à criança, que essa temática seja considerada em sua formação do profissional (ENIOLA e EVARTS, 2017).

Nesta e outras pesquisas (SILVA et al., 2018; SANTOS et al., 2016), observou-se falhas nos registros dos casos notificados de violência contra crianças, especialmente falta de preenchimento de inúmeras informações. Tal fato sinaliza a necessidade de intervenções que visem aprimorar e qualificar ações de vigilância que incluam, além da detecção precoce e oportuna dos casos, a qualidade dos dados registrados (SANTOS et al., 2016) e o funcionamento em rede de setores de proteção à criança, tais como serviços de saúde e conselhos tutelares (SANTOS et al., 2019; LIRA et al., 2018).

É válido pontuar que muitos avanços foram alcançados no enfrentamento da violência, mas muitos desafios ainda persistem, demonstrando que esse fenômeno, em suas distintas formas de expressão, deve tornar-se parte da agenda prioritária do setor saúde, conforme demonstram sua magnitude e impactos na qualidade de vida da população (MINAYO et al., 2018).

Considerando que a notificação é uma das principais etapas no processo de enfrentamento da violência infantil, visto que a partir dela derivam ações no âmbito das redes de atenção e proteção, voltadas para a promoção, prevenção de reincidências e estabelecimento de uma linha de cuidado às crianças em situação de violência (EGRY et al., 2018; TONMYR et al., 2018), apresenta-se como limitação dessa pesquisa o alto número de fichas de notificação em que não houve registro adequado das informações, a exemplo, as variáveis local de ocorrência da agressão e vínculo/grau de parentesco do agressor com a criança. Tal aspecto pode comprometer a interpretação dos fatores que corroboram à violência, bem como mascará-la, prejudicando a implementação de estratégias efetivas para seu enfrentamento.

\section{CONCLUSÃO}

Conclui-se que a violência contra criança no município investigado variou de acordo com o sexo e faixa etária, e que a família e pessoas conhecidas apresentaram-se como principais perpetradoras da violência infantil, que comumente ocorre no próprio domicílio, sugerindo que esse agravo à saúde está presente na rotina familiar e configura-se em fenômeno culturalmente aceito e negligenciado em nossa sociedade.

Visando contribuir para o enfrentamento da violência e fortalecimento da rede de proteção da criança, sugere-se que diversos atores sociais, tais como profissionais da saúde, educação e justiça, implementem atividades educativas que contemplem temas relacionados à cultura de paz e à responsabilidade da família e sociedade na construção de ambientes que oportunizem pleno crescimento e desenvolvimento infantil.

Independente de qual seja o tipo de agressão infantil, qualquer desrespeito à criança configura-se em violação dos direitos humanos e em agravo evitável, que deve ser contemplado pelas políticas públicas objetivando, especialmente, a prevenção da ocorrência e reincidência, bem como redução dos danos físicos e psicossociais que podem afetar as crianças.

Conclui-se, também, que há falhas no preenchimento das fichas de notificação da violência infantil no município, indicando, assim, a necessidade urgente de capacitar os profissionais de saúde em relação à importância desse instrumento para se implementar políticas e estratégias direcionadas às reais necessidades e vulnerabilidades das crianças e famílias. 
O desconhecimento do real quantitativo de casos de violência contra criança e de suas características pode dificultar a realização de ações capazes de interromper o círculo de violência e comprometer o enfrentamento desse agravo à saúde, contribuindo para manutenção das iniquidades sociais as quais essa população está exposta.

Todos os autores declararam não haver qualquer potencial conflito de interesses referente a este artigo.

\section{REFERÊNCIAS}

AFIFI, T. O.; FORTIER, J.; MACMILLAN, H. L.; GONZALEZ, A.; KIMBER, M.; GEORGIADES, K. et al. Examining the relationships between parent experiences and youth self-reports of slapping/spanking: a population-based cross-sectional study. BMC Public Health, v. 19, n. 1345, p. 1-8, 2019.

AUSTIN, A. Association of Adverse Childhood Experiences with Life Course Health and Development. N C Med J, v. 79, n. 2, p. 99-103, mar./apr. 2018.

BERTHOLD, O.; HOFFMANN, U.; CLEMENS, V.; WITT, A.; FEGERT, J. M. Kinderschutz im Gesundheitswesen verbessern: Fachberatung, Weiterbildung und Forschung am Beispiel des misshandlungsbedingten Kopftraumas.

Bundesgesundheitsblatt - Gesundheitsforschung Gesundheitsschutz, v. 62, n. 8, p. 960-969, 2019.

BRANQUINHO, I. D.; LANZA, F. M. Child health in primary care: evolution of brazilian policies and nurses' performance. Revista de Enfermagem do Centro-Oeste Mineiro, v. 8, p. 1-8, 2018.

BRASIL. Ministério da Saúde. Centro de Informações Estratégicas em Vigilância em Saúde - CIEVS. 2007. Disponível em: http://bvsms.saude.gov.br/bvs/publicacoes/centro_inf ormacoes_estrategicas_vigilancia_saude.pdf. Acesso em: 04/11/2019.

BRASIL. Ministério da Saúde. Sistema de Informação de Agravos de Notificação - Sinan Net. Frequência por Região de notificação segundo faixa etária, período 2014. Disponível em: http://tabnet.datasus.gov.br/cgi/tabcgi.exe?sinannet/c nv/violebr.def. Acesso em: 04/11/2019.
DESLANDES, S. F.; VIEIRA, L. J. E. S.; CAVALCANTI, L. F.; SILVA, R. M. Atendimento à saúde de crianças e adolescentes em situação de violência sexual, em quatro capitais brasileiras. Rev. Interface, Botucatu, v. 20, n. 59, p. 865-877, 2016.

EGRY, E. Y.; APOSTÓLICO, M. R.; MORAIS, T. C. P.; LISBOA, C. C. R. Enfrentar a violência infantil na Atenção Básica: como os profissionais percebem? Rev Bras Enferm, v. 70, n. 1, p. 119-125, jan./fev. 2017.

EGRY, E. Y.; APOSTOLICO, M. R.; MORAIS, T. C. P. Reporting child violence, health care flows and work process of primary health care professionals. Rev. Ciênc. saúde coletiva, v. 23, n. 1, p. 83-92, 2018.

ENIOLA, K.; EVARTS L. Diagnosis of Child Maltreatment: A Family Medicine Physician's Dilemma. South Med J., v. 110, n. 5, p. 330-336, maio 2017. DOI: 10.14423/SMJ.0000000000000644.

LIRA, S. V. G., MOREIRA, D. P.; CARNEIRO, G. M. A.; NORONHA, C. V.; VIEIRA, L. J. E. de S. Articulação entre o conselho tutelar e o setor saúde no enfrentamento à violência intrafamiliar. Trabalho, Educação e Saúde, v. 16, n. 2, p. 821-835, 2018. DOI: https://dx.doi.org/10.1590/1981-7746-sol00131.

MINAYO, M. C. S.; SOUZA, E. R.; SILVA, M. M. A.; ASSIS, S. G. Institucionalização do tema da violência no SUS: avanços e desafios. Ciênc. saúde coletiva, v. 23, n. 6, p. 2007-2016, 2018.

MOODY, G.; CANNINGS-JOHN, R.; HOOD, K.; KEMP, A.; ROBLING, M. Establishing the international prevalence of self-reported child maltreatment: a systematic review by maltreatment type and gender. BMC Public Health, v. 18, n. 1, p. 1164, out. 2018.

NAKAYA N. Mother's attributions regarding children's behavior and maltreatment. Shinrigaku Kenkyu, v. 87, n. 1, p. 40-49, 2016.

NUNES, A. J.; SALES, M. C. V. Violência contra crianças no cenário brasileiro. Ciênc. saúde coletiva, v. 21, n. 3, p. 871-880, 2016.

PLATT, V. B.; BACK, I. C.; HAUSCHILD, D. B.; GUEDERT, J. M. Violência sexual contra crianças: autores, vítimas e consequências. Rev. Ciênc. saúde coletiva, v. 23, n. 4, p. 1019-1031, 2018.

RIBEIRO, I. M. P.; RIBEIRO, A. S. T.; PRATESI, R.; GANDOLF, L. Prevalence of various forms of 
violence among school students. Rev. Acta paul. Enferm., v. 28, n. 1, p. 54-59, 2015.

SANTOS, L. F.; COSTA, M. M.; JAVAE, A. C. R. S.; MUTTI, C. F.; PACHECO, L. R. Factors that interfere with the confrontation of child violence by guardianship counselors. Saúde Debate, v. 43, n. 120, p. 137-49, 2019.

SANTOS, T. M. B. dos; CARDOSO, M. D.; PITANGUI, A. C. R.; SANTOS, Y. G. C.; PAIVA, S. M.; MELO, J. P. R.; SILVA, L. M. P. Completitude das notificações de violência perpetrada contra adolescentes em Pernambuco, Brasil. Ciênc. Saúde Colet., v. 21, n. 12, p. 39073916, 2016.

SCHEK, G.; SILVA, M. R. S.; LACHARITÉ, C.; BUENO, M. E. N. Professionals and interfamily violence against children and adolescents: in between legal and conceptual precepts. Rev Esc Enfem USP, v. 50, n. 5 , p. $779-784,2016$.

SENA, C. A. de; SILVA, M. A. da; FALBO NETO, G. H. Incidência de violência sexual em crianças e adolescentes em Recife/Pernambuco no biênio 20122013. Ciênc. saúde coletiva [online], v. 23, n. 5, p. 1591-1599, 2018. ISSN 1413-8123. Disponível em: http://www.scielo.br/scielo.php?script=sci_arttext\&pi
$\mathrm{d}=\mathrm{S} 1413-81232018000501591 \& \operatorname{lng}=\mathrm{pt} \& \mathrm{nrm}=\mathrm{iso}$. Acesso em: 04/11/2019.

SILVA, L. M. P. DA; SANTOS, T. M. B. DOS; SANTIAGO, S. R. V.; MELO, T. Q. DE; CARDOSO, M. D. Analysis of the completness of the notifications of violence perpetrated against children. Rev. enferm. UFPE on line, v. 12, n. 1, p. 91-100, jan. 2018.

TONMYR, L.; MATHEWS, B.; SHIELDS, M. E.; HOVDESTAD, W. E.; AFIFI, T. O. Does mandatory reporting legislation increase contact with child protection? - a legal doctrinal review and an analytical examination. BMC Public Health, v. 18, n. 1, p. 1021, ago. 2018.

TURNER, S.; TAILLIEU, T.; CARLETON, R. N.; SAREEN, J.; AFIFI, T. O. Association between a history of child abuse and suicidal ideation, plans and attempts among Canadian public safety personnel: a cross-sectional survey. CMAJ Open, v. 6, n. 4, p. E463-E470, 2018 DOI: 10.9778/cmajo.20170153.

UNICEF. United Nations Children's Fund. A Familiar Face: Violence in the lives of children and adolescents. 2017. Disponível em: https://www.unicef.org/publications/files/Violence_in _the_lives_of_children_and_adolescents.pdf. Acesso em: 04/11/2019. 